\title{
New generation of cancer treatment: Immunotherapy.
}

\author{
Birju M Mehta ${ }^{*}$, Vipul Kumar Patel' ${ }^{2}$, Sachin G Thakkar ${ }^{3}$ \\ ${ }^{1}$ Center for Cell and Gene Therapy, Baylor College Medicine Houston, Texas, USA \\ ${ }^{2}$ Molecular and Human Genetics, Baylor College of Medicine, Houston, Texas, USA \\ ${ }^{3}$ College of Pharmacy, Division of Molecular Pharmaceutics and Drug Delivery, The University of Texas at Austin, Austin, \\ Texas, USA
}

Accepted on October 16, 2017

\section{Editorial}

Cancer is one of the leading causes of death worldwide, accounting for 8.8 million deaths in 2015 (WHO). In 2017, there will be an estimated 1,688,780 new cancer cases diagnosed and 600,920 cancer deaths are projected to occur in the US (Siegel et al. Cancer Statistics, 2017, CA: A Cancer J Clin). The disease attacks patient's immune system and completely lowers it down. Traditional cancer treatment includes one of the following options: Chemotherapy, Radiation or Surgery. The Agency for Healthcare Research and Quality estimates that the direct medical costs for cancer in the US in 2013 were $\$ 74.8$ billion. Forty-four percent of those costs were related to the hospital outpatient services or office-based provider visits, and $40 \%$ were for inpatient hospital stays. These estimates are based on a set of large-scale surveys of families and individuals and their healthcare providers called the Medical Expenditure Panel Survey (MEPS). MEPS is the most complete, nationally representative data on healthcare use and expenditures.

Besides, the severe and life-threatening side effects are major disadvantage with traditional cancer management modalities. These treatment approaches could also lead to secondary cancer or relapse. Moreover, the heavy doses of chemo or radiation therapies could substantially increase mortality and morbidity leading to poor outcomes and sufferings.

The pioneering scientific discovery in oncology in early 90's revealed a new approach to fight against cancer through manipulation and manoeuvring the patient's own immune system. This approach is later termed as "Immunotherapy" which caught attention of physicians, scientists, drug companies, and policy makers. The immunotherapy offers several advantages over traditional treatments such as killing cancer cells with minimal side effects, better tolerability, reduced potential for mortality and morbidity, better prognosis, etc. It basically works on a simple fundamental rule of putting patient's immune system back to work and boosting it to fight the cancer antigens.

One of the crucial components of human immune system is T-cells. These cells are harvested from the patient's blood and then transformed into chimeric antigen receptors (CARs) in a specialized setting. These CARs are a type of protein that enables the T-cells to attack cancer cells. These engineered T-cells are cultured and cultivated in the lab with special care under the State of Art GMP facility to yield billions of cells. These CAR T-cells grown in laboratory are then injected into the patient's circulation. These circulating T-cells in human body fight against cancer cells and destroy them.

The current scientific discoveries focused on to finding ways to generate more powerful T-cells that will completely eliminate cancer cells from the patient's body. However, one of the main disadvantages of the immunotherapy is called "Cytokine release syndrome". The injected immune cells in the patient's body releases the cytokines involved in making the products. The patient with cytokine release syndrome develops side effects such as low blood pressure, fatigue, fever and weakness. The research studies reported that 9 out of 15 patients do not have these side effects.

Several clinical trials are in progress across the county and globe to validate use of immunotherapy in variety of cancers. Detailed information on immunotherapy clinical trials can be found from the physicians or hospitals. Also, Clinicaltrials. gov provides most updated information and availability of the trials. The patients need to pass certain eligibility criteria in order to enrol in such clinical trial. The development of such immunotherapeutic agents could take months to years before approval from FDA or other regulatory agencies for clinical use. Several completed as well as on-going clinical trials reported significant increase in survival rates with negligible side effects.

\section{*Correspondence to:}

Birju M Mehta

Center for Cell and Gene Therapy

Baylor College of Medicine

Houston, Texas

USA

Tel: (714) 526-0806

E-mail: bmehta@bcm.edu 